\title{
Diurnal variation in exercise responses in angina pectoris
}

\author{
M JOY, C M POLLARD, ^ T O NUNAN† \\ From St Peter's Hospital, Chertsey, Surrey
}

SUMMARY Thirty caucasian male patients with stable angina were investigated in two groups of nine and one group of 12 . Nine normal subjects were also studied. Patients in the first group (on no treatment) underwent symptom-limited exercise electrocardiography at 0800,1200 , and 1600 hours on the same day. Their heart rates and ST segment displacements at 1600 hours were significantly greater than at 0800 hours and the same phenomenon was seen in the second group who had been receiving propranolol $40 \mathrm{mg}$ four times a day. A similar effect was noted for ST segment displacement but not for heart rate in the third group (on no treatment) tested at 0800 hours and 1600 hours on separate days, two to three weeks apart. Normal control subjects showed no diurnal variation in heart rate and their heart rate responses at 1600 hours were reduced by propranolol. The observations show a circadian variation in the ST segment response to exercise in patients with angina and a possible training effect on heart rate with multiple exercise testing on the same day. This variation is associated with a reduction in vagal parasympathetic tone to the heart and should be taken into account in the assessment of patients with angina and in particular when comparing responses to treatment.

Circadian variation in cardiovascular variables such as the blood pressure and heart rate is well established. ${ }^{2}$ Recently, Yasue and co-workers ${ }^{3}$ described 13 patients with variant angina in whom there was an angiographically demonstrable increase in tone of the major coronary arteries in the morning when compared with the afternoon. This was associated with a significant variation in exercise tolerance. Little attention appears to have been paid to possible circadian variation in classical angina pectoris and for this reason the clinical impression that a patient's angina is worse in the morning was investigated.

\section{Subjects and methods}

Thirty caucasian male patients on no treatment with a history of stable angina pectoris for at least six months were studied in two groups of nine (A and B) and one

Based on a paper given to the European Congress of Cardiology, Paris 1980.

^Present address: St George's Hospital, London SW17 ORE. tPresent address: St Thomas's Hospital, London SE1 7EH.

Accepted for publication 23 March 1982 group of 12 (C). Nine asymptomatic normotensive $\frac{3}{\sqrt{3}}$ male volunteers were also studied (D). All the patients were normotensive (BP $<160 / 95 \mathrm{mmHg})$, had no past history of myocardial infarction, and no evidence of $\mathbb{D}$ valvular heart disease. They also had a normal chest $x$-ray film and resting electrocardiogram but on exercise using the Bruce treadmill protocol ${ }^{4}$ developed at least $1 \mathrm{~mm}$ of $\mathrm{J}$ point depression with horizontal $\stackrel{\circ}{\circ}$ depression or downslope of the ST segment. These exercise-induced changes have been associated with a $\frac{\text { ㅇ }}{5}$ $93 \%$ specificity for coronary artery disease. ${ }^{5}$ Echocar- $\rightarrow$ diography was performed to exclude left ventricular or septal hypertrophy and mitral leaflet prolapse as N possible causes for false positive exercise electrocardiographic responses. There was no significant differ- $\triangle$ ence between the mean ages of the patient groups $\omega$ $(56.17 \pm 1.71$ years overall) or their mean weights? (75.67 $\pm 1.72 \mathrm{~kg}$ overall), though the group D subjectso were younger $(39.39 \pm 2.6$ years, $p<0.001)$. Informed signed consent was obtained from all participants.

EXERCISE TECHNIQUES

All subjects underwent treadmill electrocardiography to angina or exhaustion. An Avionics E10 treadmill 2 was used in conjunction with an Avionics 3000 three 156 
channel oscillographic monitor. Leads CM4, CM5, and CM6 were studied, recordings being taken automatically at rest, after each minute of exercise, at maximum exercise, and at each of 10 minutes during the recovery period. The chart speed was regularly checked and the recording system optimally damped. The heart rate responses were computed and checked by measurement from the chart. ST segment displacement was noted at $60 \mathrm{~ms}$ after the nadir of the $S$ wave by two observers independently, unaware of the circumstances of the test, consensus being obtained with the third.

\section{GROUP A}

The patients in group A (on no drugs) underwent exercise electrocardiography at 0800 hours after a light breakfast, at 1200 hours, and at 1600 hours on the same day. A light lunch was taken after the midday test.

\section{GROUP B}

The patients in group B were subjected to the same procedure as group A but had received propranolol $40 \mathrm{mg}$ four times a day for at least four weeks before assessment. Drugs were taken on the day of study after each exercise recording.

\section{GROUP C}

The patients in group C (on no drugs) were randomly allocated into two subgroups. The first underwent a single exercise test at 0800 hours followed by a further single test at 1600 hours, two to three weeks later. The second subgroup started with the 1600 hours test and after two to three weeks underwent a further test at $\mathbf{0 8 0 0}$ hours. During analysis both subgroups were combined, randomisation neutralising any changes in performance which might have occurred with training.

\section{GROUP D}

The subjects in group D were asymptomatic male colleagues who were randomly allocated into two subgroups. The first subgroup of five underwent the triple procedure while taking no drugs and were retested a mean of four weeks later after receiving propranolol $40 \mathrm{mg}$ four times a day. Drugs were taken during the day of assessment as described for group B. The second subgroup of four received propranolol for at least two weeks before undergoing the triple procedure which was repeated after four weeks on no drugs.

The means and standard error (SEM) of the observations were calculated and the paired observations, that is 0800 hours vs 1600 hours, were examined using Student's paired $t$ test. This technique examines the means of the differences between the paired observations and their relation to zero.
Results

\section{GROUP A}

The heart rate responses of this group (on no treatment) are illustrated in Fig. 1. The maximum heart rate was significantly higher in the afternoon than in the morning $(p<0.025)$, in spite of comparable walking times. The one minute recovery heart rate was also higher $(p<0.025)$, but the two minute recovery value failed to achieve significance $(0.01>p>0.05)$. The ST segment displacements (Fig. 2) were greater in the afternoon both at maximum heart rate and throughout recovery $(p<0.02$ or better), with the exception of the three minute observation $(0.01>p>0.05)$. The 1200 hours observations adopted an intermediate position and, though the walking times at both 1200 hours and 1600 hours were greater than at $\mathbf{0 8 0 0}$ hours, the difference was not significant $(0.1>p>0.05)$.

\section{GROUP B}

The heart rate responses of this group (who had been receiving propranolol) are shown in Fig. 3. The maximum heart rate was significantly higher in the afternoon $(p<0.005)$ in spite of the drug, and this difference was good until the end of the third minute of recovery $(p<0.025)$. Likewise (Fig. 4) the ST segment displacements were greater in the afternoon at maximum exercise $(p<0.005)$ and after one minute of

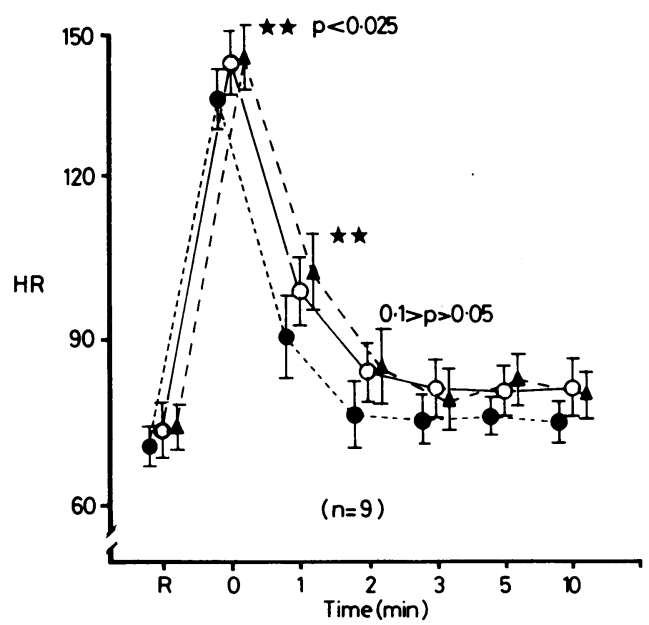

Fig. 1 Symptom-limited exercise heart rate responses of the group A patients; $R$ (rest); $O$ (maximum heart rate); $1,2,3,5$, 10 (minutes of recovery); $H R$ (heart rate). The closed circles represent the means of the 0800 hour values, the open circles those of the 1200 hour values, and the triangles those of the 1600 hour values. The bars represent 1 SEM. The 1600 heart rates are significantly higher at maximum exercise and at one minute of recovery. 


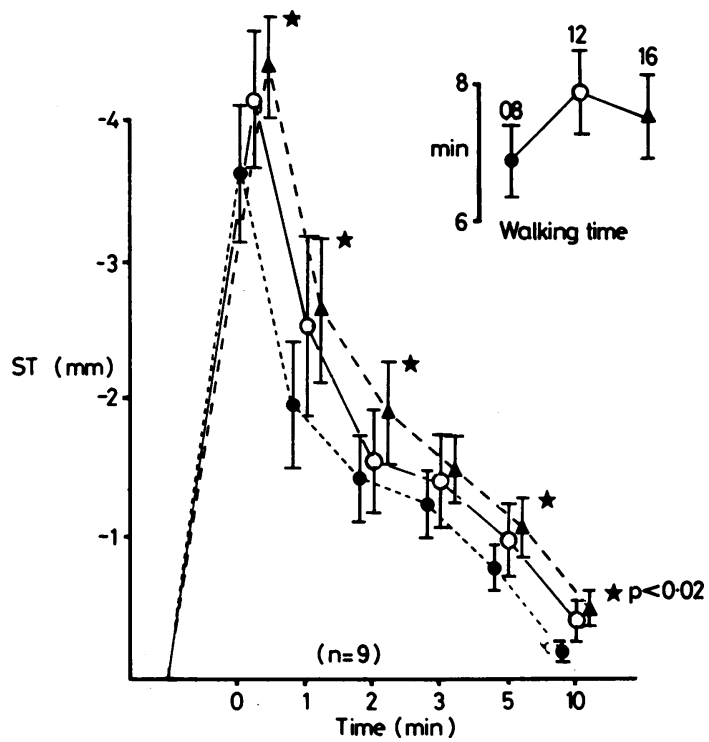

Fig. $2 S T$ segment responses of the group $A$ patients illustrated in Fig. 1; the symbols are as for Fig. 1 , the ordinate representing millimetres of ST segment depression. The 1600 hours $S T$ segment displacements are significantly greater at maximum heart rate and throughout recovery apart from the third minute.

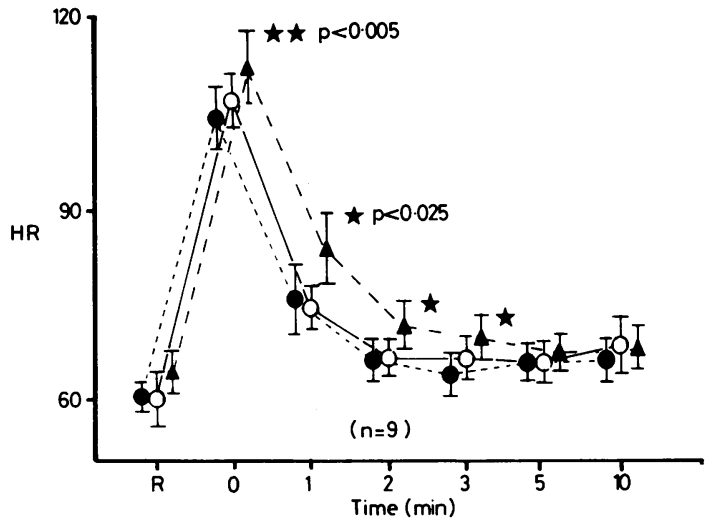

Fig. 3 Symptom-limited exercise heart rate responses of the group B patients; the symbols are as for Fig. 1. The 1600 hours heart rates are significantly higher at maximum exercise and for the first three minutes of recovery in spite of beta blockade.

recovery $(p<0.05)$, the differences thereafter being not significant. No significant difference was seen in the walking times.

\section{GROUP C}

The heart rate responses of this group (on no treatment) are illustrated in Fig. 5. No significant differences were seen $(p<0 \cdot 15)$. The ST segment displace- ments observed (Fig. 6) were significantly greater both at maximum heart rate and throughout recovery $(p<0.05$ or better) at 1600 hours when compared with 0800 hours.

\section{GROUP D}

The heart rate responses of this group when taking no treatment are illustrated in Fig. 7. No significant differences were seen. In sharp contrast to the patients with angina, the afternoon heart rate responses (Fig. 8) were lower when the group received propranolol ( $p<0.025$ for maximum heart rate through five minutes' recovery; $\mathrm{p}<0.05$ at 10 minutes).

\section{Discussion}

The pathophysiology of ST segment displacement during acute myocardial ischaemia remains uncertain. Angina pectoris in patients with ischaemic heart disease occurs at a reproducible value of rate pressure product $^{6}$ in response to exercise and of tension-time index in response to atrial pacing. ${ }^{7}$ Exercise-induced ischaemic ST segment depression correlates closely with indices expressing myocardial oxygen consumption (derived from heart rate, blood pressure, and ejection time), but the best single determinant is heart rate $^{89}$ which was thus used in this study. Nevertheless, variability in exercise tolerance with dislocation of the ST segment/heart rate relation has been described in continued sustained exercise, ${ }^{10}$ and with training, ${ }^{11}$ and brief severe exercise may be associated with greater triple products at the point of angina than after more limited exercise. ${ }^{12}$ Detry et al. ${ }^{13} 14$ sub-

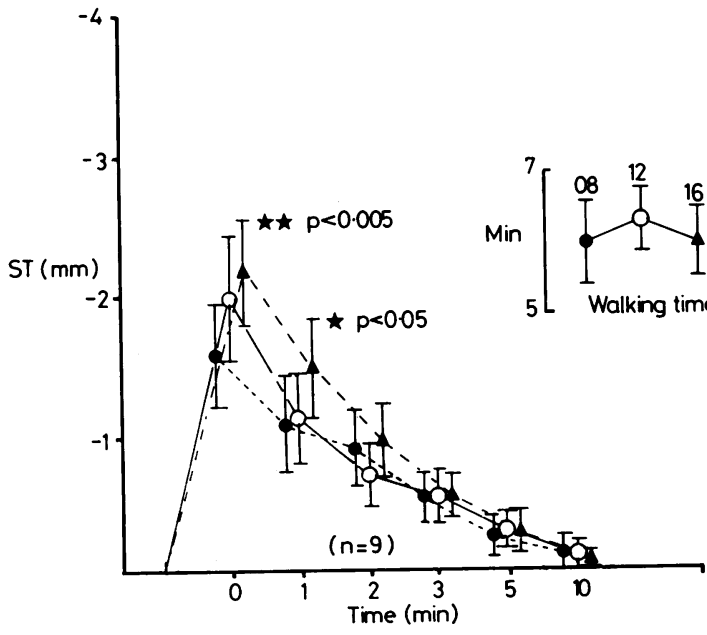

Fig. $4 S T$ segment responses of the group $B$ patients illustrated in Fig. 3; the symbols are as for Fig. 1 and 2. The 1600 hours $S T$ segment displacements are significantly greater at maximum heart rate and during the first minute of recovery. 


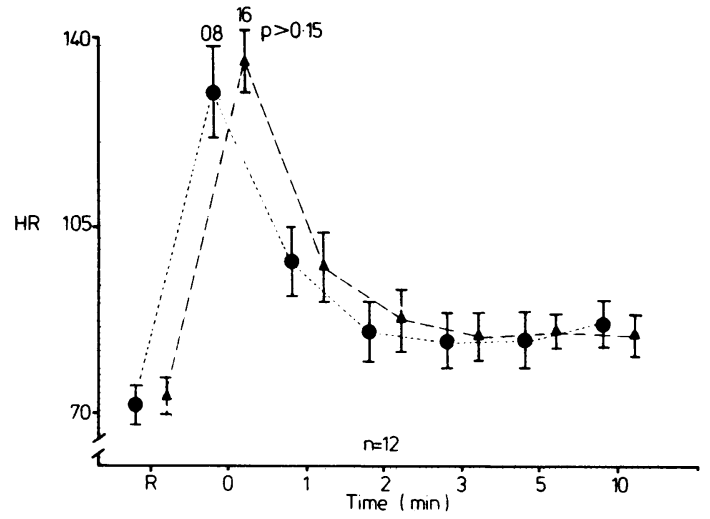

Fig. 5 Symptom-limited exercise heart rate responses of the group $C$ patients exercised on one occasion on each of two days, two to three weeks apart; the symbols are as for Fig. 1 . No significant difference was observed between the pairs of observations at any point.

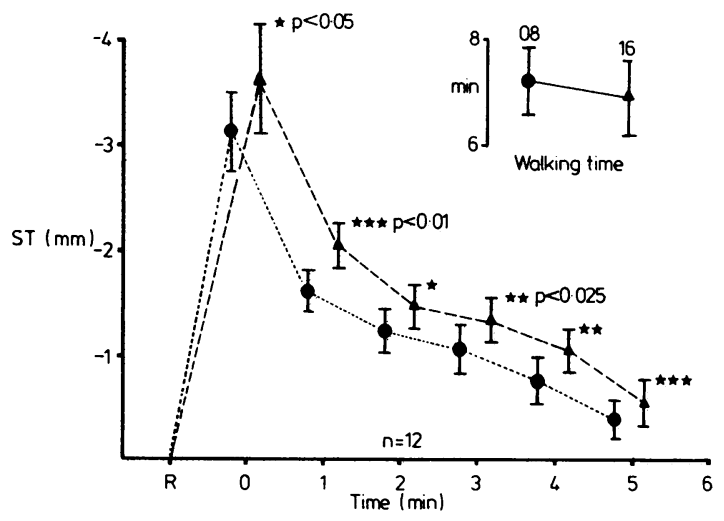

Fig. $6 S T$ segment displacements of the group $C$ patients illustrated in Fig. 5; the symbols are as for Fig. 1 and 2. The 1600 hours $S T$ segment displacements are sigmificantly greater at maximum heart rate and throughout recovery.

jected patients with angina to a three month training routine and observed a significant decrease in heart rate and pressure rate product at submaximal exercise, but at maximal exertion these values were greater and associated with increased ST segment displacement. These investigators suggested that training could raise the threshold for angina. Sim and Neill ${ }^{15}$ also confirmed a higher threshold for angina induced by exercise after training but not during atrial pacing. The heart rate and ST segment observations in group $A$ are equally consistent with an exercise-induced functional adaptation or with a circadian variation in exercise performance. The group $C$ patients who underwent a single 0800 hours exercise test followed by a further test two to three weeks later showed grea- ter ST segment displacements at 1600 hours, suggesting a circadian variation in tolerance of myocardial ischaemia if ST segment displacement reflects myocardial oxygen deficit and the pathogenesis of pain. ${ }^{8}$ The heart rate response at 1600 hours in group

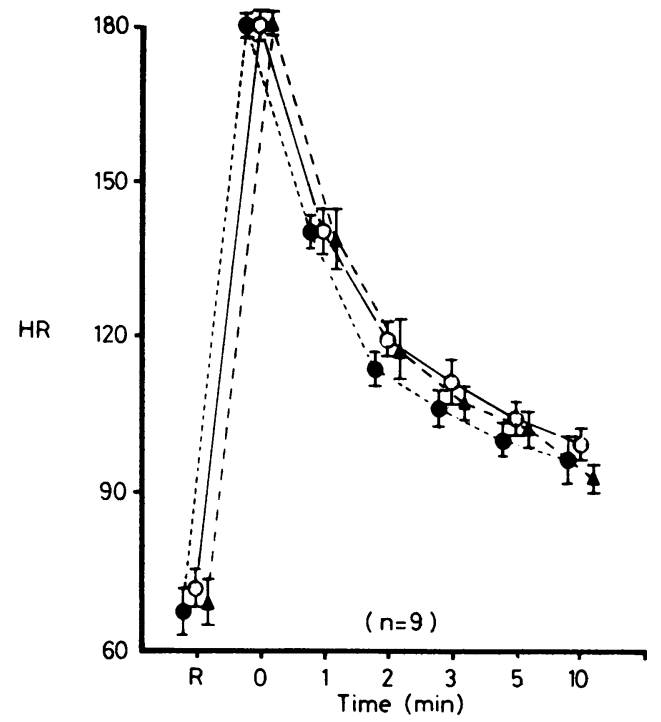

Fig. 7 Symptom-limited exercise heart rate responses of the normal subjects in group $D$, on no treatment; the symbols are as for Fig. 1. No diumal variation is seen.

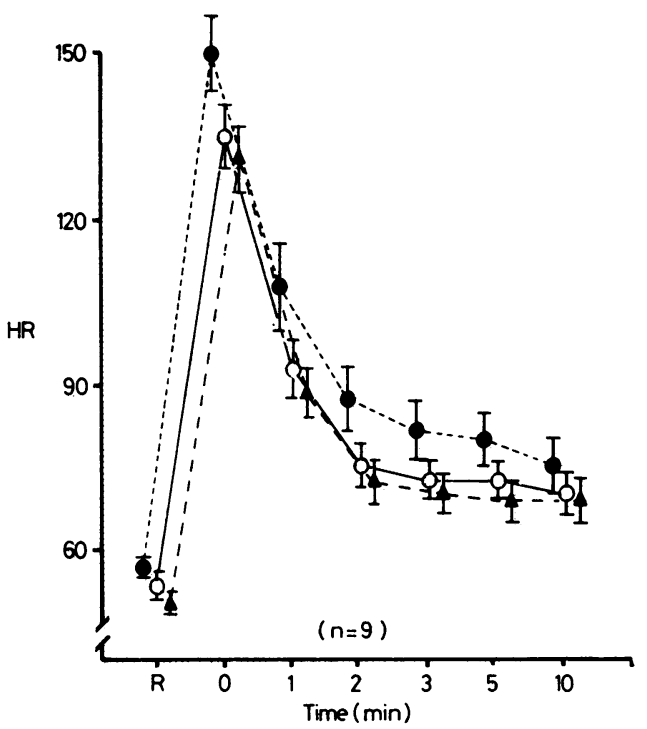

Fig. 8 Symptom-limited exercise heart rate responses of the normal subjects in group $D$ illustrated in Fig. 7 after two to four weeks' treatment with propranolol $40 \mathrm{mg} q \mathrm{ds}$; the symbols are as for Fig. 1. The 1600 hour heart rates are significantly lower than the 0800 hour heart rates at maximum exercise and throughout recovery. 
A, not shown by group C, may be explained by a training dependent adaptation ${ }^{15}$ which does not occur in normal subjects off treatment (Fig. 7). The pattern of enhanced heart rate responses and ST segment displacements seen in group A was also seen in group B, in spite of treatment with propranolol (Fig. 3, 4), though this beta adrenoceptor blocking agent reduced the heart rate response of the normal subjects (Fig. 8), as would be expected.

It may be inferred that the normal subjects receiving propranolol (Fig. 8) have a reduced heart rate response to exercise because of reduction in sympathetic drive to the heart, vagal parasympathetic withdrawal being complete at maximal exercise. In contrast, the higher heart rate responses at 1600 hours in group B can only be explained on the basis of a withdrawal of vagal tone unless the unlikely circumstance of sympathetic overdrive is proposed. The circadian variation in ST segment displacement and the exercise dependent adaptation of the heart rate are thus associated with or mediated by a reduction in vagal tone to the heart. Yasue et al. ${ }^{3}$ showed a circadian variation in exercise capability in patients with variant angina, and using angiographic techniques convincingly showed an increase in tone of the major coronary arteries in the morning, compared with the afternoon. Though there is evidence that metabolic effects $^{16}$ and local oxygen concentration ${ }^{17}$ are prime determinants of coronary artery tone, evidence for neurogenic influence is available, ${ }^{18}$ which may involve the parasympathetic nervous system. ${ }^{19}$ It is therefore interesting to note that the variation in exercise responses in subjects with angina observed above is associated with or mediated by changes in parasympathetic tone to the heart. Such variations in exercise response have implications when exercise electrocardiography is used to monitor patient progress or study responses to treatment.

\section{References}

1 Brooks H, Carroll JH. A clinical study of the effects of sleep and rest on blood pressure. Arch Intern Med 1912; 10: 97-102.

2 Smyth HS, Sleight P, Pickering GW. Reflex regulation of arterial pressure during sleep in man. Circ Res 1969; 24: 109-21.

3 Yasue $\mathrm{H}$, Omote S, Takizawa A, Nagao M, Miwa K, Tanaka S. Circadian variation of exercise capacity in patients with Prinzmetal's angina: role of exerciseinduced coronary arterial spasm. Circulation 1979; 59: 938-48.

4 Doan AE, Peterson DR, Blackmon JR, Bruce RA. Myocardial ischemia after maximal exercise in healthy men. Am Heart f 1965; 69: 11-21.
5 Goldschlager N, Seltzer A, Cohn K. Treadmill stress tests as indicators of presence and severity of coronary artery disease. Ann Intern Med 1976; 85: 277-86.

6 Robinson BF. Relation of heart rate and systolic blood pressure to the onset of pain in angina pectoris. Circulation 1967; 35: 1073-83.

7 Sowton GE, Balcon R, Cross D, Frick MH. Measurement of the angina threshold using atrial pacing. A new technique for the study of angina pectoris. Cardiovasc Res 1967; 1: 301-7.

8 Detry JMR, Piette F, Brasseur LA. Hemodynamic determinants of exercise ST segment depression in coronary patients. Circulation 1970; 42: 593-9.

9 Nelson RR, Gobel FL, Jorgensen CR, Wang K, Wang $\mathrm{Y}$, Taylor HL. Hemodynamic predictors of myocardial oxygen consumption during static and dynamic exercise. Circulation 1974; 50: 1179-89.

10 MacAlpin RN, Kattus AA. Adaptation to exercise in angina pectoris: the electrocardiogram during treadmill walking and coronary angiographic findings. Circulation 1966; 33: 183-201.

11 Raffo JA, Luksic IY, Kappagoda CT, Mary DAGS, Whitaker W, Linden RJ. Effects of physical training on myocardial ischaemia in patients with coronary artery disease. Br Heart F 1980; 43: 262-9.

12 Redwood DR, Rosing DR, Goldstein RE, Beiser GD, Epstein SE. Importance of the design of an exercise protocol in the evaluation of patients with angina pectoris. Circulation 1971; 43: 618-28.

13 Detry JMR, Rousseau M, Vandenbroucke G, Kusumi F, Brasseur LA, Bruce RA. Increase arteriovenous oxygen difference after physical training in coronary heart disease. Circulation 1971; 44: 109-18.

14 Detry JMR, Bruce RA. Effects of physical training on exertional ST segment depression in coronary heart disease. Circulation 1971; 44: 390-6.

15 Sim DN, Neill WA. Investigation of the physiological basis for increased exercise threshold for angina pectoris after physical conditioning. $\mathcal{f}$ Clin Invest 1974; 54: 76370.

16 Yasue H, Nagao M, Omote S, Takizawa A, Miwa K, Tanaka S. Coronary arterial spasm and Prinzmetal's variant form of angina induced by hyperventilation and Tris-buffer infusion. Circulation 1978; 58: 56-62.

17 Gellai M, Norton JM, Detar R. Evidence for direct control of coronary vascular tone by oxygen. Circ Res 1973; 32: 279-89.

18 Berne RM, De Geest H, Levy MN. Influence of the cardiac nerves on coronary resistance. Am $\mathcal{f}$ Physiol 1965; 208: 763-9.

19 Yasue H, Touyama M, Shimamoto M, Kato H, Tanaka $S$, Akiyama F. Role of autonomic nervous system in the pathogenesis of Prinzmetal's variant form of angina. Circulation 1974; 50: 534-9.

Requests for reprints to Dr Michael Joy, St Peter's Hospital, Guildford Road, Chertsey, Surrey KT16 0PZ. 